\title{
Antimicrobial Sensitivity Pattern of Microorganisms Isolated from Vaginal Infections at a Tertiary Hospital in Bangalore, India
}

Nagalakshmi Narayana-Swamy, ${ }^{1}$ Padmasri Ramalingappa, ${ }^{1}$ Urvashi Bhatara.

\begin{abstract}
Background: The vagina contains dozens of microbiological species in variable quantities and is, therefore, considered a complex environment. Among the microorganisms, bacteria have important repercussions on women's health. The present study was conducted to elucidate this type of vaginal isolates and their sensitivity towards currently used antibiotics. Methods: This was a retrospective study conducted at the Department of Obstetrics and Gynaecology, Sapthagiri Hospital, Bangalore, India from January 2012 to December 2013. All symptomatic women who had a high vaginal swab taken for culture and sensitivity testing were included in this study. Antibiotic susceptibility was tested using disc diffusion method (modified Kirby-Bauer's method). The antibiotic sensitivity patterns of isolated microorganisms were studied. Results: Out of 200 patients, $95 \%$ had positive vaginal cultures. Fifteen types of microorganisms were isolated. The highest frequency of infection was seen at the age of 20-30 years, followed by 41-50 years and 31-40 years, and a low frequency of infection was observed above 50 years of age. The most prevalent pathogen was Escherichia coli, followed by Streptococcus agalactiae and diphtheroids with equal incidence. Among the antibiotics tested, isolated pathogens were completely resistant to nalidixic acid and highly sensitive to meropenem and imepenem. Conclusion: The high prevalence of gynaecological infections demands that patients with symptoms undergo thorough investigation with cultures and sensitivity essays. Changes in treatment protocols are required to treat vaginal infections effectively.
\end{abstract}

Keywords: Vaginitis, Microbiota, Anti-Bacterial Agents, Microbial Sensitivity Tests (Source: MeSH-NLM).

About the Author: Ms. Nagalakshmi Narayana Swamy is a third year medical student of Sapthagiri Institute of Medical Sciences and Research Center Bangalore, India of a five and a half year programme.
Submission: Dec 18, 2014

Acceptance: Mar 31, 2015

Publication: Mar 31, 2015

Process: Peer-reviewed

\section{Introduction}

The vagina is a complex ecosystem containing a variety of micro-organisms. ${ }^{1}$ This unique environment undergoes significant changes throughout life, from birth to puberty and menopause. ${ }^{2}$ Females are more prone to urinary and vaginal infections because of the anatomical and functional proximity to the anal canal and due to the short urethra. ${ }^{3}$ The sex steroid hormones play a vital role in stabilizing this environment. In normal women, the estrogen accounts for the maturation of vaginal epithelium, resulting in the accumulation of glycogen which helps in the maintenance of vaginal $\mathrm{pH}^{4}{ }^{4}$

The causative organisms can be endogenous, iatrogenic or sexually transmitted. The human body harbours hundreds of organisms of gram-positive and gram-negative varieties in the lower one-third of vagina. A key protective role is played by the lactic acid-producing bacteria in keeping the vaginal pH low. ${ }^{5}$

Common organisms are Neisseria gonorrhoea, Trichomonas vaginalis, Streptococcus agalactiae (group B Streptococcus) and Chlamydia trachomatis. Presentation includes itching and pain in the external genitalia and vagina, painful sexual intercourse, and the presence of abnormal vaginal discharge. ${ }^{6}$

The balanced vaginal environment varies with practices like douching, dressing, use of contraceptives and sexual activi- ty.? Coexistent factors like diabetes and pregnancy also play a role in the vaginal ecosystem imbalance. Surprisingly, not many studies have investigated the prevalence of vaginal infections in relation to age and that of the antibiotic sensitivity pattern. ${ }^{8}$ Morbidity associated with these infections also affects the economic productivity and quality of life of many individual women and consequently of communities as a whole.

Many women believe that such infections are normal and part of the female experience and do not seek care due to shame or lack of information. ${ }^{9}$ In the pregnant woman, these infections lead to preterm labour, chorioamnionitis, premature rupture of membranes and low birth weight of the neonate, leading to high perinatal mortality. ${ }^{10}$

These infections can be easily detected by simple tests such as a vaginal swab for culture and sensitivity testing, which inform us the causative organism as well the antibiotic to use against it. " As per the Centre for Disease Control and Prevention (CDC) guidelines, the management includes therapy based on susceptibility pattern, partner notification, follow-up and health promotion. The aim of our study was to identify the common organisms in vaginal infections and to discuss the sensitivity pattern based on culture sensitivity. By studying sensitivity pattern to antibiotics in these strains, we may revise the recommendations for treatment protocol in such patients.

${ }^{1}$ Sapthagiri Institute of Medical Sciences and Research Center, Bangalore, India 


\section{Methods}

This is a retrospective study conducted at Department of Obstetrics and Gynaecology, Sapthagiri Hospital (a tertiary health care centre), Bangalore from January 2012 to December 2013. Two hundred women aged between 20 to 65 years who had presented with symptoms of vaginal infections were included based on the previous records of culture and sensitivity obtained from the Department of Microbiology. They were labelled with a unique identifier to ensure confidentiality and freedom from bias. All the women had presented with one or more of the following symptoms: vaginal discharge, dyspareunia, malodour, dysuria, itching and fever. Those using antibiotics during the last two weeks and those with a recent history of vaginal instrumentation were excluded from the study.

Three swabs were collected for each woman. The first sample was a high vaginal swab from which culture was performed using blood agar for gram-positive bacteria, MacConkey agar for gram-negative bacteria (especially the Enterobacteriaceae), and Sabouraud's dextrose agar for fungi. They were incubated for 48 hours. The second sample was used for direct microscopic examination of Trichomonas vaginalis. The cervical samples were collected in suspected cases only and processed for the detection of Chlamydia trachomatis. Once the samples were obtained, they were transported to the Microbiology Laboratory Department at Sapthagiri Hospital, Bangalore.

Identification of microorganisms: isolated bacteria were identified using conventional methods, including colonial morphology, Gram stain, motility, germ tube test and biochemical tests (DNAse, catalase, Indole, coagulase, lactose fermentation, urease, oxidase, sugar fermentation, citrate utilization). Identification of Streptococci was based on haemolysis and thereby categorized according to Lancefield's grouping.

Antibiotic susceptibility was tested using disc diffusion method (modified Kirby-Bauer's method). Antimicrobials tested for sensitivity were amikacin, ampicillin, amoxicillin, imepenem, meropenem, ceftazidime, ceftriaxone, tigecycline, ciprofloxacin, cefpodoxime, nalidixic acid and cefixime.

Data obtained were presented as distribution of microorganisms with respect to age, number and percentage of patients from which the microorganisms were isolated, and antimicrobial sensitivity patterns. Analysis was done using Microsoft Excel program. However, the analysis was done with respect to age only, as details of social factors were not available. Based on data obtained, observations were drawn regarding the age-specific infective rates and present status of antimicrobial sensitivity patterns.

The ethical approval for this study was given by the Ethics and Research Committee of Sapthagiri Institute of Medical Sciences and Research Centre, Bangalore.

\section{Results}

Out of 200 women enrolled in the study, $95 \%$ had positive vaginal cultures. Fifteen microorganisms, including gram-negative bacteria, were isolated. The highest infection rate was observed among women aged between 20 and 30 years $(39.5 \%)$, followed by those aged $31-40$ years $(19.0 \%)$ and $41-50$ years $(31.0 \%)$, and the lowest frequency of infection was observed among those aged between 51 and 65 years (5.5\%), as shown in Table 1 .
Table 1. Distribution of Microorganisms with Respect to Age and Infection Rates.

\begin{tabular}{|c|c|c|}
\hline $\begin{array}{l}\text { Age } \\
\text { (years) }\end{array}$ & Frequency (\%) & Organisms \\
\hline $20-30$ & $79(39.5 \%)$ & $\begin{array}{l}\text { Commensals, other gram-negative bacteria, } \\
\text { extended-spectrum beta-lactamase-produ- } \\
\text { cing Escherichia coli, coagulase-negative Sta- } \\
\text { phylococcus aureus, diphtheroids, Candida } \\
\text { albicans, Acinetobacter. }\end{array}$ \\
\hline $31-40$ & $38(19.0 \%)$ & $\begin{array}{l}\text { Commensals, Escherichia coli, Streptococcus } \\
\text { agalactiae, Gardnerella vaginalis, Klebsiella } \\
\text { pneumoniae, Citrobacter. }\end{array}$ \\
\hline $41-50$ & $62(31.0 \%)$ & $\begin{array}{l}\text { Proteus vulgaris, Escherichia coli, Klebsiella } \\
\text { pneumoniae, commensals, Micrococci, me- } \\
\text { thicillin-resistant Staphylococcus aureus. }\end{array}$ \\
\hline$>50$ & $11(5.5 \%)$ & $\begin{array}{l}\text { Escherichia coli, Enterococci, Klebsiella pneu- } \\
\text { moniae, commensals, Pseudomonas aerugi- } \\
\text { nosa, Proteus vulgaris, coagulase-negative } \\
\text { Staphylococcus aureus. }\end{array}$ \\
\hline
\end{tabular}

Table 2. Types and Proportions of Microorganisms Recovered from Women with Vaginal Infections at Department of Obstetrics and Gynaecology, Sapthagiri Hospital, Bangalore.

\begin{tabular}{|lc|}
\hline Organism & Frequency $(\%)$ \\
\hline Escherichia coli & $23(11.5 \%)$ \\
\hline $\begin{array}{l}\text { Extended-spectrum beta-lactamase-producing Esche- } \\
\text { richia coli }\end{array}$ & $13(6.5 \%)$ \\
\hline Streptococcus agalactiae & $12(6.0 \%)$ \\
\hline Diphtheroids & $12(6.0 \%)$ \\
\hline Pseudomonas aeruginosa & $10(5.0 \%)$ \\
\hline Methicillin-resistant Staphylococcus aureus & $10(5.0 \%)$ \\
\hline Gram-negative bacteria & $10(5.0 \%)$ \\
\hline Cardnerella vaginalis & $10(5.0 \%)$ \\
\hline Citrobacter & $10(5.0 \%)$ \\
\hline Enterococci & $8(4.0 \%)$ \\
\hline Proteus vulgaris & $8(4.0 \%)$ \\
\hline Coagulase-negative Staphylococcus aureus & $8(4.0 \%)$ \\
\hline Micrococci & $7(3.5 \%)$ \\
\hline Klebsiella pneumoniae & $7(3.5 \%)$ \\
\hline Acinetobacter & $7(3.5 \%)$ \\
\hline Candida albicans & $5(2.5 \%)$ \\
\hline Mixed growth & $2(1.0 \%)$ \\
\hline
\end{tabular}

Microorganism with the highest frequency of infection was Escherichia coli ( $11.5 \%)$, followed by extended-spectrum beta-lactamase-producing Escherichia coli (6.5\%), diphtheroids (6.0\%), Streptococcus agalactiae (6.0\%), Pseudomonas aeruginosa $(5.0 \%)$, Gardnerella vaginalis $(5.0 \%)$, methicillin-resistant Staphylococcus aureus (MRSA) (5.0\%), Citrobacter $(5.0 \%)$, and other gram-negative bacteria (5.0\%). Candida albicans was isolated in $2.5 \%$ of the cases. Other organisms, such as Proteus vulgaris, coagulase-negative Staphylococcus aureus, Acinetobacter, Klebsiella pneumoniae, Micrococci and Enterococci, were also isolated (Table 2). 
Figure 1. Antibiotic Sensitivity Pattern of the Isolated E. Coli, Proteus, Diphtheroids, Streptococci, Micrococci, Pseudomonas and Enterococci to 11 Antimicrobial Agents.

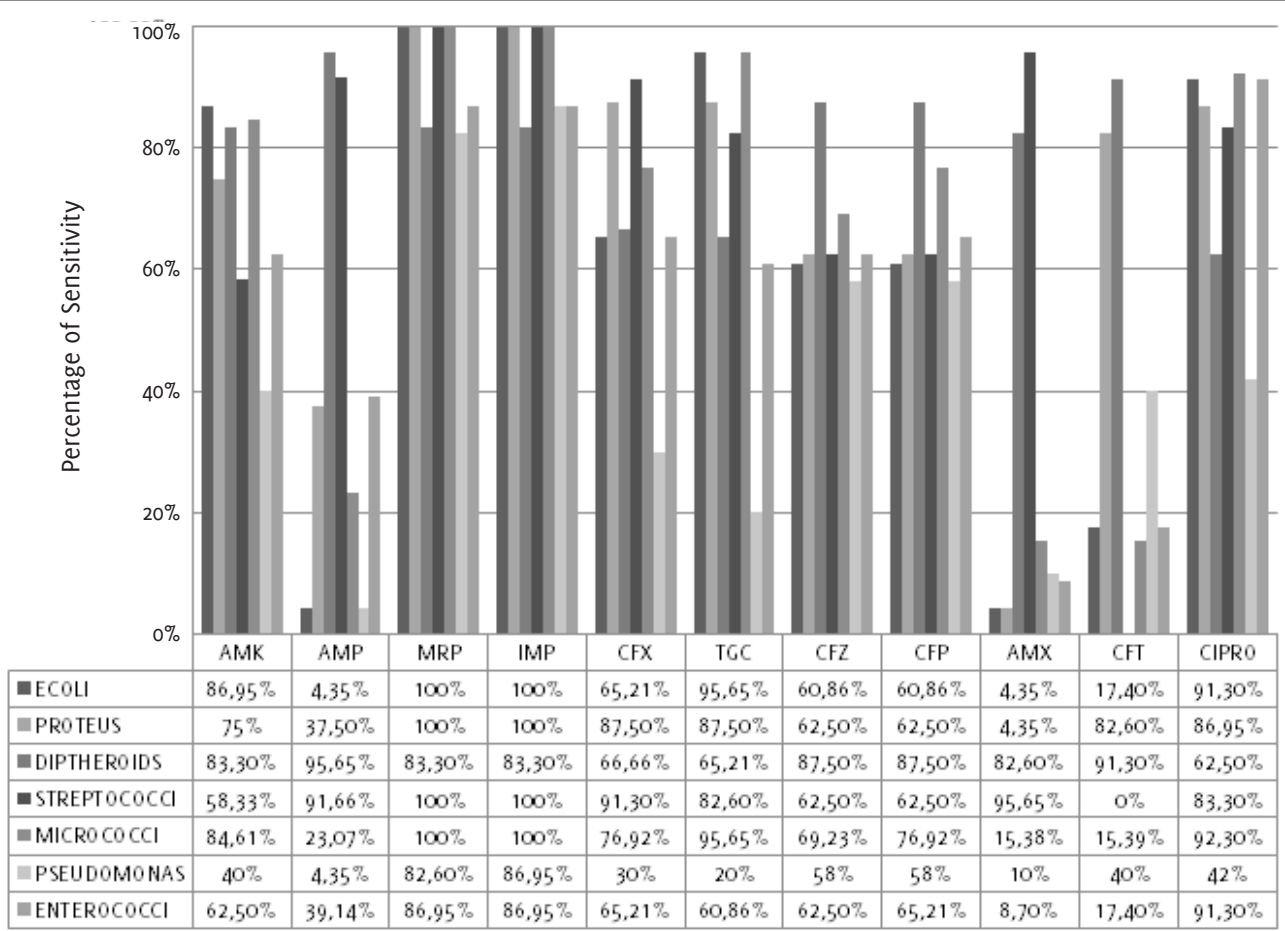

Legend: ECOLI-Escherichia coli. Antibiotics: AMK-Amikacin, AMP-Ampicillin, MRP-Meropenem, IMP-Imipenem, CFX-Cefixime, TGC-Tigecycline, CFZ-Ceftazidime, CFP-Cefpodoxime, AMX-Amoxicillin, NA-Nalidixic acid, CFT-Ceftriaxone, CIPRO-Ciprofloxacin.

Figure 2. Antibiotic Sensitivity Pattern of the Isolated Coagulase-Negative Staphylococcus Aureus, Acinetobacter, Citrobacter, Klebsiella, ESBL-producing E. Coli, Gardnerella Vaginalis, MRSA and other GNB to 11 Antimicrobial Agents.

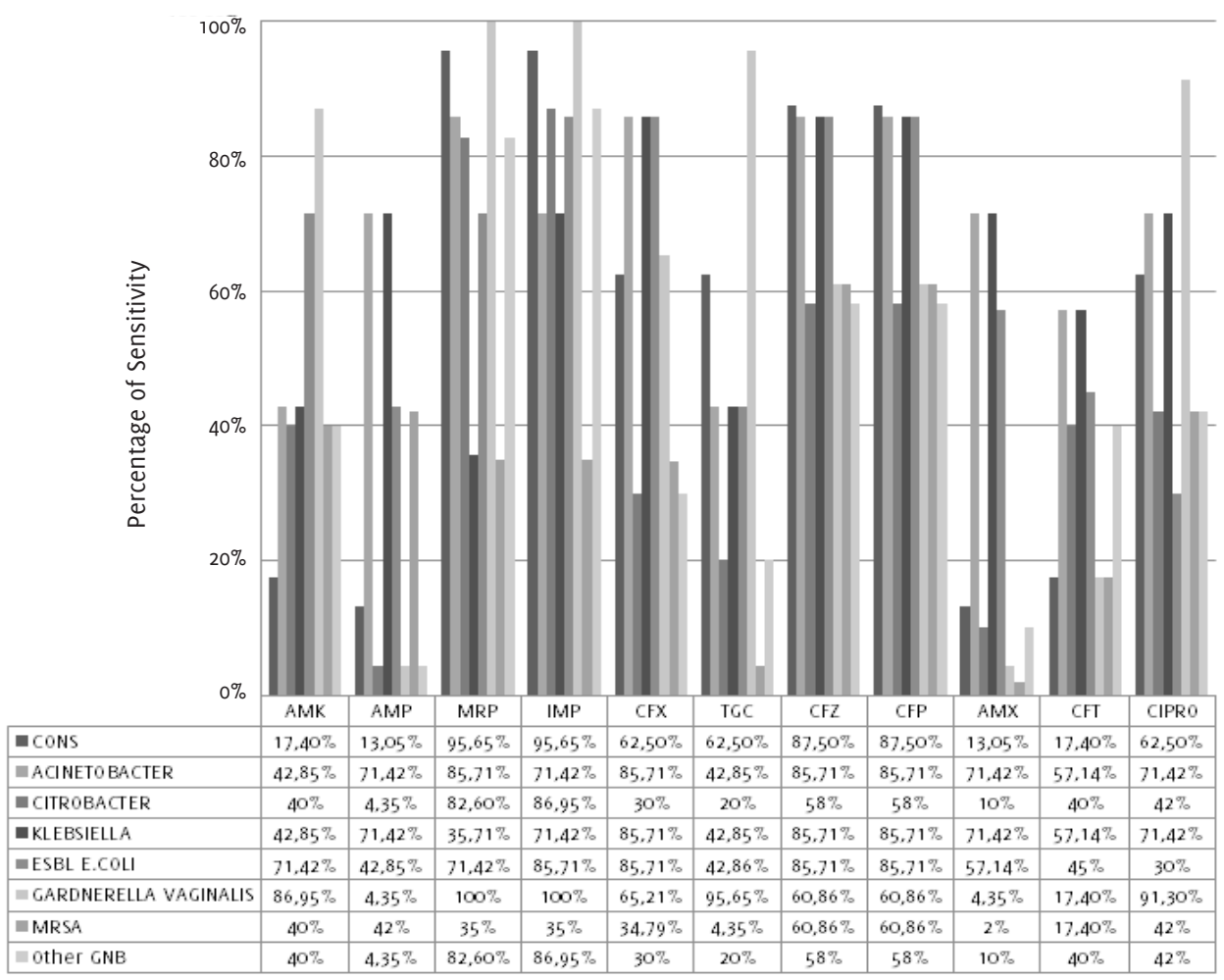

Legend: CONS-Coagulase-negative Staphylococcus aureus, ESBL E.COLI-Extended-spectrum beta-lactamases-producing Escherichia coli, MRSA-Methicillin-resistant Staphylococcus aureus, GNB-Gram-negative bacteria. Antibiotics: AMK-Amikacin, AMP-Ampicillin, MRP-Meropenem, IMP-Imipenem, CFX-Cefixime, TGC-Tigecycline, CFZ-Ceftazidime, CFP-Cefpodoxime, AMX-Amoxicillin, NA-Nalidixic acid, CFT-Ceftriaxone, CIPRO-Ciprofloxacin. 
Table 3. Antimicrobial Sensitivity Pattern of Isolated Microorganims.

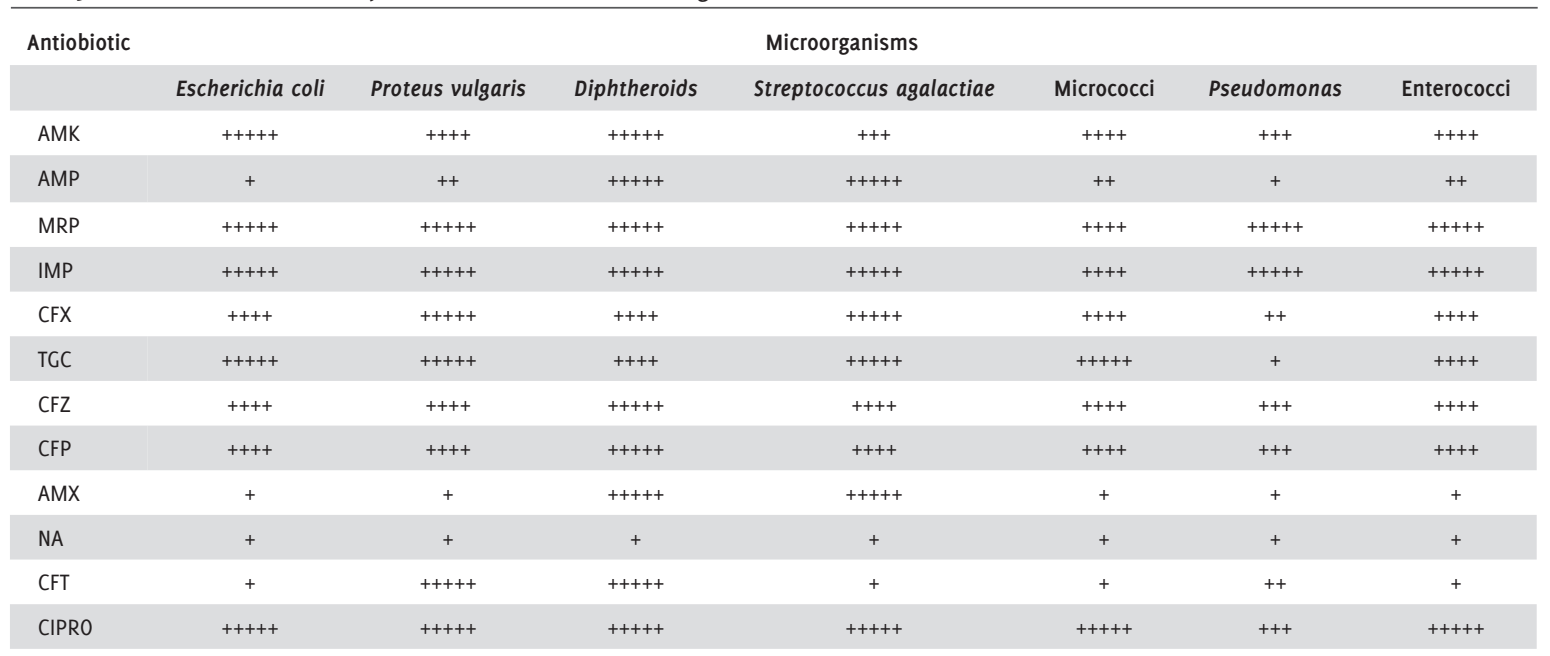

Legend: Legend: Sensitivity rates: $80 \%-100 \%$ : +++++, 60\%-80\%: ++++, 40\%-60\%: +++, 20\%-40\%: ++, 0\%-20\%: +. Antibiotics: AMK-Amikacin, AMP-Ampicillin, MRP-Meropenem, IMP-Imipenem, CFX-Cefixime, TGC-Tigecycline, CFZ-Ceftazidime, CFP-Cefpodoxime, AMX-Amoxicillin, NA-Nalidixic acid, CFT-Ceftriaxone, CIPRO-Ciprofloxacin.

Table 4. Antimicrobial Sensitivity Pattern of Isolated Microorganims.

\begin{tabular}{|c|c|c|c|c|c|c|c|c|}
\hline Antiobiotic $^{\dagger}$ & \multicolumn{8}{|c|}{ Microorganisms ${ }^{\ddagger}$} \\
\hline AMK & + & +++ & +++ & +++ & ++++ & +++++ & +++ & +++ \\
\hline AMP & + & ++++ & + & ++++ & +++ & + & +++ & + \\
\hline MRP & +++++ & +++++ & +++++ & ++ & ++++ & +++++ & ++ & +++++ \\
\hline IMP & +++++ & ++++ & +++++ & ++++ & +++++ & +++++ & ++ & +++++ \\
\hline TGC & ++++ & +++ & + & +++ & +++ & +++++ & + & + \\
\hline CFZ & +++++ & +++++ & +++ & +++++ & +++++ & ++++ & ++++ & +++ \\
\hline CFP & +++++ & +++++ & +++ & +++++ & +++++ & ++++ & ++++ & +++ \\
\hline AMX & + & ++++ & + & ++++ & +++ & + & + & + \\
\hline
\end{tabular}

Legend: Legend: Sensitivity rates: $80 \%-100 \%$ : +++++, 60\%-80\%: $++++, 40 \%-60 \%:+++, 20 \%-40 \%:++, 0 \%-20 \%:+{ }^{+}$AMK-Amikacin, AMP-Ampicillin, MRP-Meropenem, IMP-Imipenem, CFX-Cefixime, TGC-Tigecycline, CFZ-Ceftazidime, CFP-Cefpodoxime, AMX-Amoxicillin, NA-Nalidixic acid, CFT-Ceftriaxone, CIPRO-Ciprofloxacin. ${ }^{\ddagger}$ CoNS-Coagulase-negative Staphylococci, ESBL-Extended-spectrum beta-lactamase, MRSA-Methicillin-resistant Staphylococcus aureus, GNB-Gram-negative bacilli.

Escherichia coli (Figure 1 and Table 3) was most sensitive to meropenem (100\%) and imipenem (100\%) and most resistant to amoxicillin $(4.4 \%)$. Proteus vulgaris showed sensitivity to meropenem (100\%), imipenem $(100 \%)$, cefixime $(87.5 \%)$, tigecycline $(87.5 \%)$ and ciprofloxacin $(87.0 \%)$. Diphtheroids showed sensitivity to ampicillin $(95.7 \%)$, ceftriaxone $(91.3 \%)$, cefpodoxime $(87.5 \%)$, ceftazidime $(87.5 \%)$, and others. Streptococcus agalactiae showed sensitivity to meropenem (100\%), imipenem (100\%), amoxicillin $(95.7 \%)$, ampicillin $(91.7 \%)$ and cefixime (91.3\%). Micrococci showed $100 \%$ sensitivity to meropenem and imipenem. Pseudomonas aeruginosa and Enterococci were sensitive to meropenem and imipenem, as shown in Figure 1 and Table 3. Complete resistance to nalidixic acid was noted with all organisms.

As shown in Figure 2 and Table 4, coagulase-negative Staphylococcus aureus showed sensitivity to meropenem (95.7\%), imipenem $(95.7 \%)$, cefpodoxime $(87.5 \%)$, ceftazidime $(87.5 \%)$, cefixime $(62.5 \%)$ and tigecycline $(62.5 \%)$. Acinetobacter showed $85.7 \%$ sensitivity to meropenem, cefixime and cefpodoxime; $\mathrm{Ci}$ - trobacter and other gram-negative bacteria showed sensitivity to imipenem (87.0\%) and meropenem (82.6\%). Klebsiella pneumoniae showed sensitivity to ceftazidime and cefpodoxime. Extended-spectrum beta-lactamase-producing Escherichia coli showed sensitivity to imipenem and cefixime, whereas Gardnerella vaginalis showed sensitivity to meropenem (100\%) and imipenem (100\%). MRSA strains were sensitive to ceftazidime $(60.9 \%)$ and cefpodoxime (60.9\%).

\section{Discussion}

Vaginal infections have wide implications for women's health, being the most common gynaecological problem..$^{2}$ It is believed that the lactobacilli play an important role in maintaining normal vaginal ecosystem and preventing the growth of opportunistic bacteria. ${ }^{33}$ Our study demonstrates the prevalence of potential vaginal pathogens in symptomatic women.

The results of our study are comparable to the study by Lakshmi $\mathrm{K}$ et al., which compared the prevalence of vaginal infections between premenopausal and postmenopausal women. ${ }^{14}$ 
Increased infections in post-menopausal women are due to the vagina being colonised by pathogenic organisms more than the protective organisms. ${ }^{15}$ The highest frequency of infection was noted at an age of 20 to 30 years with a fall in the frequency of infection as age advanced. Similar results with respect to age were seen in a Kenyan study, although it was limited to the study of only one organism. ${ }^{16}$

Several microorganisms were isolated in our study, and those with the highest frequencies were Escherichia coli, diphtheroids, Klebsiella pneumoniae, Streptococcus agalactiae, Pseudomonas aeruginosa, Proteus vulgaris, MRSA, Micrococci, Enterococci, Acinetobacter, Citrobacter and Candida albicans. Commensal growths were found in $14 \%$ of cases that need not be treated, but necessary measures such as identification of risk factors (douching, sprays, diabetes) and their prevention have to be carried out.

We also found Escherichia coli to be the most common pathogenic bacteria (11.5\%) isolated from culture. In fact, Escherichia coli was found to be the most prevalent pathogen isolated not only from high vaginal swabs but also urine, pus, blood and wounds, as seen in a study conducted by Dutta $S$ et al., in Dhaka. ${ }^{17}$

Candida albicans is tolerant to the acidic environment and is hence found in the vagina, but the concentrations are too low to cause symptoms. In conditions of decreased local immunity, the hyphae would multiply and transform into infective patterns that result in symptomatic vaginitis. ${ }^{18}$ Colonization of Candida species also happens during pregnancy, resulting in symptomatic vaginitis. ${ }^{19}$

Infections with MRSA became a global health issue in 1960s, when the strains were first identified. They may be acquired nosocomially or from the community. What make them difficult to treat are their multiple antibiotic resistant profiles and wide varying prevalence. ${ }^{20}$ One should keep in mind that higher antibiotics may be required to treat these infections.

In our study, $6 \%$ of the women carried Streptococcus agalactiae (Group B Streptococci). However, information regarding their pregnancy status is not available. Maternal group B Streptococci (CBS) colonisation is a major risk factor for GBS disease in neonates. ${ }^{21}$ In pregnant women, GBS causes cystitis, amnionitis, endometritis and stillbirth; occasionally, it leads to endocarditis or meningitis.
Coagulase-negative Staphylococci (CoNS), which are considered to be skin commensals, were found in $4 \%$ of the cases. No cases of trichomoniasis, Chlamydia infection, and Neisseria gonorrhoeae infection were noted.

The presence of co-morbidities like hospitalization, immunosuppression and co-existent reproductive tract infections have to be evaluated accordingly. It is known that vaginal infections, due to a disruption of normal vaginal flora, increase the risk of sexually transmitted infection, especially human immunodeficiency virus (HIV). ${ }^{22}$ However, our study did not identify any association with HIV, and diabetic status records were not available. No patients had taken hormone supplements or any other medications that could interfere with the results.

Our study has several limitations. The practice of swab culture is done mostly in clinical microbiological laboratories, and clinical diagnosis may be suboptimal. Only regularly used antibiotics were included in the sensitivity testing, and socio-demographic factors have not been considered. These limitations have to be overcome by future studies, and proper practices have to be implemented in order to preserve these lifesaving drugs for the future.

Diagnosis of these infections based on culture sensitivity is a definite step in treatment of these infections. In regular practice, fixed protocols are followed. Inadequate treatment with antimicrobials due to non-compliance or under the prescription of drugs results in high incidence of recurrence. Extensive resistance rates have emerged among commonly used antibiotics due to indiscriminate use. Newer antibiotics like imipenem and meropenem are highly effective but expensive. ${ }^{23}$ Short term effects of antimicrobial regimens have been tested and proved through clinical trials to be effective in achieving clinical and microbiological cure. Newer therapeutic approaches include the development of new drugs, phage therapy (bacterial viruses can be robust anti-bacterial agents in vitro), photodynamic inactivation of micro-organisms and immunomodulators. ${ }^{24} \mathrm{~A}$ significant proportion of pathogens causing vaginal infections are resistant to the conventionally used antibiotics. This study is a step in familiarizing sensitivity and resistance patterns to used antibiotics, preventing resistance and thus preventing the chronic sequelae. Thus, we raise a question of changing the syndromic protocol to treatment protocol based on culture sensitivity. Substantial health gains with a reduction of the disease burden among women should be the long term goal of treatment which should be intended with knowledge of culture sensitivity. 


\section{References}

1. Larsen B, Monif GR. Understanding the bacterial flora of the female genital tract. Clin Infect Dis. 2001 Feb 15;32(4):e69-77.

2. Romero R, Hassan SS, Gajer P, Tarca AL, Fadrosh DW, Nikita L, et al. The composition and stability of the vaginal microbiota of normal pregnant women is different from that of non-pregnant women. Microbiome. 2014 Feb 3;2(1):4.

3. Puri R, Malhotra J. Recurrent urinary tract infection (UTI) in women. South Asian Federation of Obstetrics and Gynecology. 2009 Jan-Apr;1(1):10-3.

4. Lehman R. Clinical approach to recognizing and managing a patient with vaginal atrophy: a guide for physician assistants. Internet Journal of Academic Physician Assistants. 2009;8(1).

5. Ravel J, Gajer P, Abdo Z, Schneider CM, Koenig SS, McCulle SL, et al. Vaginal microbiome of reproductive-age women. Proc Natl Acad Sci U S A. 2011 Mar;108(Suppl 1):4680-7.

6. Ahmed HM, Rasool VH, Al-Tawil NG. Prevalence of abnormal vaginal signs and symptoms among attendees of Obstetrics and Gynecology Department of Shaqlawa Hospital. Medical Journal of Babylon. 2014;11(1):120-9.

7. Jejeebhoy $S$, Koenig $M$, Elias $C$, editors. Investigating reproductive tract infections and other gynaecological disorders: a multidisciplinary research approach. Cambridge (UK): Cambridge University Press; 2003. p. 30-81.

8. Karou SD, Djigma F, Sagna T, Nadembega C, Zeba M, Kabre A, et al. Antimicrobial resistance of abnormal vaginal discharges microorganisms in 0uagadougou, Burkina Faso. Asian Pac J Trop Biomed. 2012 Apr;2(4):294-7.

9. Bergström S. Infection-related morbidities in the mother, fetus and neonate. J Nutr. 2003 May;133(5 Suppl 2):1656S-1660S.

10. Aubyn GB, Tagoe DN. Prevalence of vaginal infections and associated lifestyles of students in the University of Cape Coast, Chana. Asian Pac J Trop Dis. 2013 Aug;3(4):267-70.

11. Owen MK, Clenney TL. Management of vaginitis. Am Fam Physician. 2004 Dec 1;70(11):2125-32.

12. Khan SA, Amir F, Altaf S, Tanveer R. Evaluation of common organisms causing vaginal discharge. J Ayub Med Coll Abbottabad. 2009 Apr-Jun;21(2):90-3. 13. Shipitsyna E, Roos A, Datcu R, Hallén A, Fredlund $H$, Jensen JS, et al. Composition of the vaginal microbiota in women of reproductive age-sensitive and specific molecular diagnosis of bacterial vaginosis is possible? PLOS ONE. 2013 Apr 9;8(4):e60670
14. Lakshmi K, Chitralekha S, Illamani V, Menezes G A. Prevalence of bacterial vaginal infections in pre and postmenopausal women. Int J Pharm Bio Sci. 2012 0ct;3(4):(B)949-56.

15. Cauci S, Driussi S, De Santo D, Penacchioni P, Iannicelli T, Lanzafame P, et al. Prevalence of bacterial vaginosis and vaginal flora changes in peri- and postmenopausal women. J Clin Microbiol. 2002 Jun;40(6):2147-52.

16. Kohli R, Konya WP, Obura T, Stones W, Revathi G. Prevalence of genital Chlamydia infection in urban women of reproductive age, Nairobi, Kenya. BMC Res Notes. 2013 Feb 4;6:44.

17. Dutta S, Hassan MR, Rahman F, Jilani MS, Noor R. Study of antimicrobial susceptibility of clinically significant microorganisms isolated from selected areas of Dhaka, Bangladesh. Bangladesh Journal of Medical Science. 2013 Ian 1;12(1):34-42.

18. Witkin SS, Linhares IM, Giraldo P. Bacterial flora of the female genital tract: function and immune regulation. Best Pract Res Clin Obstet Gynaecol. 2007 Jun;21(3):347-54.

19. Shrestha S, Tuladhar NR, Basnyat S, Acharya CP, Shrestha P, Kumar P. Prevalence of vaginitis among pregnant women attending Paropakar Maternity and Women's Hospital, Thapathali, Kathmandu, Nepal. Nepal Med Coll J. 2011 Dec;13(4):293-6.

20. David MZ, Daum RS. Community-associated methicillin-resistant Staphylococcus aureus: epidemiology and clinical consequences of an emerging epidemic. Clin Microbiol Rev. 2010 Jul;23(3):616-87.

21. Buchan BW, Faron ML, Fuller D, Davis TE, Mayne D, Ledeboer NA. Multicenter clinical evaluation of the Xpert GBS LB assay for detection of group B Streptococcus in prenatal screening specimens. J Clin Microbiol. 2015 Feb;53(2):443-8.

22. Balkus JE, Richardson BA, Mandaliya K, Kiarie J, Jaoko W, Ndinya-Achola J0, et al. Establishing and sustaining a healthy vaginal environment: analysis of data from a randomized trial of periodic presumptive treatment for vaginal infections. J Infect Dis. 2011 Jul 15;204(2):323-6.

23. Patel J, Bhatt J, Javiya V, Patel K. Anti-microbial susceptibility patterns of Enterobacteriaceae isolated from a tertiary care unit in Gujarat. Internet Journal of Microbiology. 2008;6(1).

24. Fuchs BB, Tegos GP, Hamblin MR, Mylonakis E. Susceptibility of Cryptococcus neoformans to photodynamic inactivation is associated with cell wall integrity. Antimicrob Agents Chemother. 2007 Aug; 51(8):2929-36.

\section{Acknowledgments}

Our sincere gratitude to Dr Padmasri Ramalingappa, Professor and Head of Department of Obstetrics and Cynecology, and Dr Urvashi Bhatara, Assistant Professor of Obstetrics and Gynecology at Sapthagiri Institute of Medical Sciences and Research Center, for their continuous support and inspiration throughout the work.

\section{Conflict of Interest Statement it Funding}

The Authors have no funding, financial relationships or conflicts of interest to disclose.

\section{Author Contributions}

Conception and design the work/idea, Contribution of patients or study material: PR, UB. Collect data/obtaining results, Write the manuscript: NNS. Analysis and interpretation of data, Critical revision of the manuscript: NNS, PR, UB. Statistical advice: UB.

Cite as:

Narayana-Swamy N, Ramalingappa P, Bhatara U. Antimicrobial Sensitivity Pattern of Microorganisms Isolated from Vaginal Infections at a Tertiary Hospital in Bangalore, India. Int J Med Students. 2014 Nov-2015 Mar;3(1):34-9. 